\title{
Benefits of Exergy-Based Analysis for Aerospace Engineering Applications_-Part I
}

\author{
John H. Doty, ${ }^{1}$ José A. Camberos, ${ }^{2}$ and David J. Moorhouse ${ }^{2}$ \\ ${ }^{1}$ Department of Engineering Management \& Systems, The University of Dayton, Dayton, OH 45469, USA \\ ${ }^{2}$ Multidisciplinary Science \& Technologies Center, U.S. Air Force Research Laboratory, Wright-Patterson AFB, OH 45433, USA
}

Correspondence should be addressed to John H. Doty, john.doty@notes.udayton.edu

Received 11 March 2009; Revised 15 September 2009; Accepted 23 October 2009

Recommended by Victor Giurgiutiu

This paper compares the analysis of systems from two different perspectives: an energy-based focus and an exergy-based focus. A complex system was simply modeled as interacting thermodynamic systems to illustrate the differences in analysis methodologies and results. The energy-based analysis had combinations of calculated states that are infeasible. On the other hand, the exergybased analyses only allow feasible states. More importantly, the exergy-based analyses provide clearer insight to the combination of operating conditions for optimum system-level performance. The results strongly suggest changing the analysis/design paradigm used in aerospace engineering from energy-based to exergy-based. This methodology shift is even more critical in exploratory research and development where previous experience may not be available to provide guidance. Although the models used herein may appear simplistic, the message is very powerful and extensible to higher-fidelity models: the 1st Law is only a necessary condition for design, whereas the 1st and 2nd Laws provide the sufficiency condition.

Copyright ( 92009 John H. Doty et al. This is an open access article distributed under the Creative Commons Attribution License, which permits unrestricted use, distribution, and reproduction in any medium, provided the original work is properly cited.

\section{Introduction}

Aerospace vehicle design has historically been conducted in stages of development [1]. These stages typically involve engine development, airframe development, avionics \& control development, and then some form of lifecycle maintenance and optimization after-the-fact. This approach has saved money in the past as components and systems were reused (leveraged) from one system into another (e.g., using the same, or nearly the same, engine in more than one aircraft.). We will refer to this approach as "ad-hoc". However, this approach does not necessarily produce the optimum system performance for an aerospace vehicle. In fact, after integration of all the subsystems into an overall system, the ad-hoc approach often introduces a performance penalty for each subsystem compared to its "stand-alone" performance. Thus, the overall system performance may be quite different than the stand-alone subsystem's performance may suggest.

Newer design methodologies based upon exergy, entropy, and availability concepts are in various stages of development and implementation [2-4]. However, these approaches, in general, are not well accepted by the engineering and design community at-large due to the sometimes nebulous terminology and often ill-suited use of example problems for data validation. Furthermore, many of these newer approaches lack relevance to the aerospace community $[5,6]$. It is therefore the motivation of this work to present the following via simplified analyses.

\section{The governing equations of a system, represented by the conservation laws, are viewed as the necessary conditions for the system. However, the governing equations must be supplemented by the 2nd Law as the sufficient condition in order to ensure process, and therefore, system feasibility.}

Note 1. The simplified model presented herein may be replaced by a higher-fidelity model, but the interpretation is the same-we must employ necessary and sufficient conditions for feasible analysis and design.

A methodology of analysis is presented herein, based upon combined energy and entropy principles (1st and 2 nd 
Laws), that is compared to a traditional methodology that employs energy principles only (1st Law). We refer to this unified approach as the exergy method, which combines the 1st and 2nd Laws of thermodynamics into a general balance equation representing the useful work loss for a given system or process. We selected a generic system-of-systems to enable semianalytical results for benchmark validation studies in order to focus on the interpretation of the results, rather than the complexity of the solution methodology. Also, simplified analyses were performed using only one variable (one degree-of-freedom, $1 \mathrm{DOF}$ ) in order to provide easier interpretation of the results. Additional variables may be added in a straightforward manner in order to emulate more complex systems. However, the understanding gained from the simpler systems translates directly into more complex systems. Just as importantly, the insights gained from the exergy-based analysis provide the basis for more sophisticated analysis, design, and optimization.

The goal of the simplified systems approach is to illustrate the influence of component losses such as heat transfer on overall system performance when viewed only from energy balance, 1st Law perspective (conservation of energy) compared to a combined exergy destruction perspective. The analyses were performed using the standard conservation Laws (1st Law focus) $[7,8]$ as well as methodology incorporating exergy destruction (combined 1st and 2nd Laws) [911]. The results, although presented for a simplified system, clearly demonstrate the utility of the exergy-based analyses compared to 1st Law-only analyses.

\section{Background}

In order to analyze the design of a flight vehicle in the context of this paper, different aspects need to be considered. A certain quantity of work is always required to accomplish the mission; there are also some unavoidable loss (irreversibility, e.g., due to fuel combustion) and lastly system losses that can be reduced or eliminated by design optimization. Systemlevel losses occur in many forms and negatively impact performance. Typical system losses in aerospace vehicles include friction, heat transfer, sudden compressions (e.g., shock waves), fluid mixing, electrical resistance, inelastic solid deformations, and chemical reactions [11]. Most of these losses are characterized in their own unique way and use different units of measure. More importantly, these losses are characterized or scaled locally and such characterization often does not readily translate to the system level. Capturing system losses locally in terms of standard 1st Law methodologies (energy-based) does not promote a systemslevel analysis and awareness for consistent comparisons. The combined exergy-based approach, however, does enable a consistent systems-level interpretation of losses (exergy destruction) that makes integration of components into systems more logical and intuitive [12-14].

Representing component losses, however they may occur, in a consistent system-level manner is desirable. For example, heat transfer losses are typically reported in kilowatts $(\mathrm{kW})$, whereas friction losses are often reported as total pressure drop in kilopascals (kPa) [7] or reported as a drag force in aerodynamic analyses. This difference in "book keeping" makes it difficult to compare system-level impacts of changes in heat transfer and/or friction. For example, a $1 \%$ change in heat transfer has a different impact on system performance than a $1 \%$ change in pressure drop. However, capturing the heat transfer and friction losses in terms of exergy destruction enables the losses to be represented in the same manner. As such, a 1\% exergy destruction resulting from heat transfer losses has the same impact as a $1 \%$ exergy destruction resulting from frictional losses. This consistent reporting methodology enables truer and simpler comparisons of integrated performance based upon minimization of overall system losses rather than focusing on individual component losses. In this fashion, a truly integrated approach is possible because regardless of where or how the losses occur, system performance is penalized accordingly. The focus is then on integrated system performance not on component optimization [15-18].

Parametric exploratory analyses and preliminary designs are often performed in terms of energy-based methods (1st Law focus) without foreknowledge of the results ( $a$ priori investigation). In such studies, variables of interest are permuted through ranges of heretofore unknown operating conditions and the resulting combinations of variables are analyzed in terms of component and then system performance. Consider such a methodology employing only heat transfer rate as the variable of interest and turbine performance as the metric for performance. Varying heat transfer rate by a few percent relative to some baseline establishes a range of conditions for which turbine performance is to be predicted. Establishing ad-hoc conditions in this manner provides no guarantee that the parametric combination of variables provides a physically possible state for system operation. In other words, it is possible, and indeed likely, when performing exploratory parametric combinations of variables that the output variable (turbine performance) may not be physically realistic. In other words, the predicted turbine performance may not satisfy the second-Law principle stipulating nonnegative entropy generation. This 1stLaw only methodology therefore makes integration and trade studies difficult to compare when performing trades studies and preliminary design.

Contrast the 1st Law methodology for analysis and preliminary design just described with a combined 1st and 2nd Law methodology that is exergy-based. Consider the same scenario and test matrix as before, with only heat transfer rate as the variables of interest. However, using the combined 1st and 2nd Law methodology, the heat transfer rate is recast in terms of exergy destruction that provides a consistent quantification metric for assessment (i.e., a common "currency") at the system-level. In such a fashion, any losses, regardless of how or where they may occur, may be translated directly ("rolled up") to the systemlevel. Thus, system analysis, design, and comparisons are more readily performed. Additionally, the combined exergybased methodology, by invoking the second-Law principle, ensures the feasibility of the process physically. In other words, if the parametric combination of variables produces 


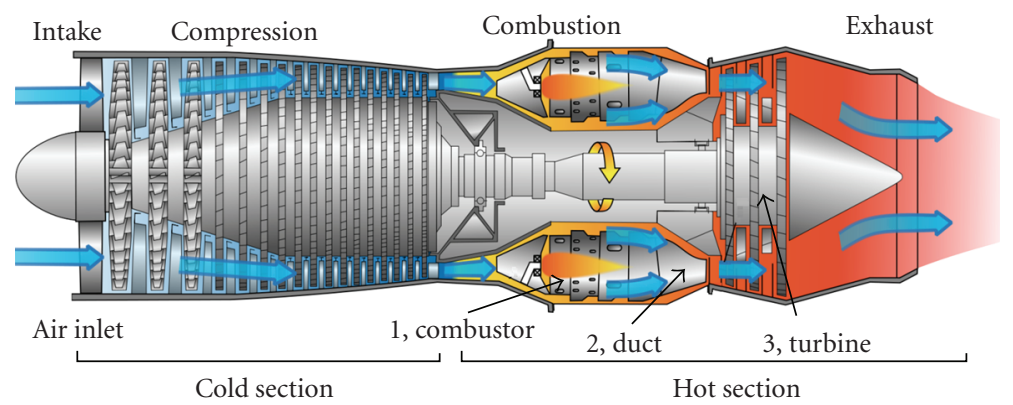

FIGURE 1: Sketch of complex turbojet-based engine outlining focus areas for simplified analysis. (System 1: Combustor as energy source, System 2: Duct as energy transfer, System 3: Turbine as energy conversion.). Source: http://commons.wikimedia.org/wiki/File:Jet_engine. svg

TABLE 1: Description of simplified internal flow systems.

\begin{tabular}{lll}
\hline System no. & Description & Relevance \\
\hline 1 & $\begin{array}{l}\text { Energy source: modeled as a system delivering } \\
\text { super-heated steam to represent combustion and } \\
\text { compression process }\end{array}$ & Represents inlet, compressor, and combustor systems \\
& $\begin{array}{l}\text { Energy transfer: modeled as a duct connecting System 1 } \\
\text { to System 3, with heat transfer possible } \\
\text { Energy conversion: modeled as a system converting } \\
\text { energy from super-heated steam to work }\end{array}$ & $\begin{array}{l}\text { Represents combustor and diffuser (or similar device) } \\
\text { connecting combustor to turbine }\end{array}$ \\
\hline
\end{tabular}

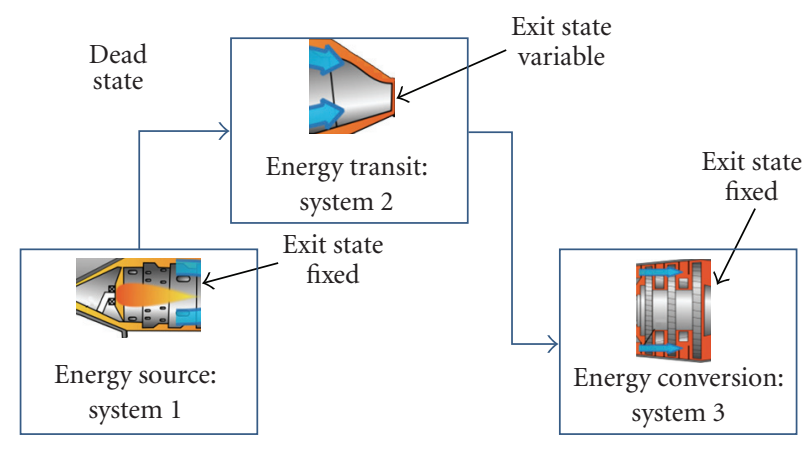

FIGURE 2: Sketch of simplified internal flow system that represents a more complex system component of a turbojet engine.

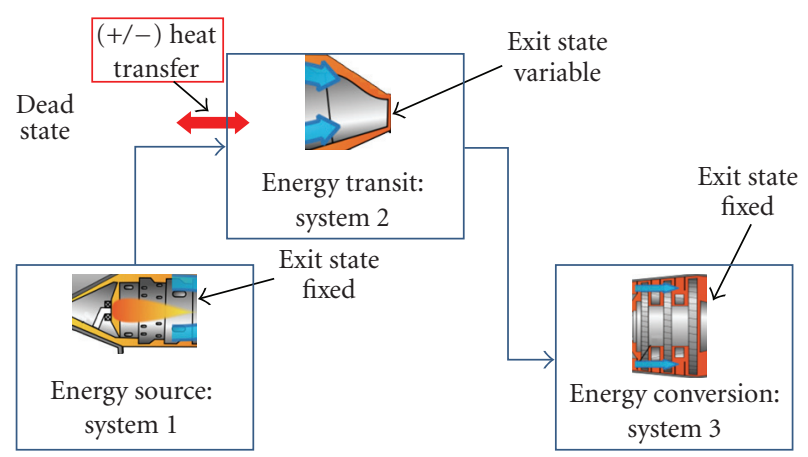

FIgURE 3: Sketch of simplified internal flow system illustrating possibility of heat transfer in System 2 (pipe). System 1 is assumed to operate at fixed conditions, while System 3 operates at fixed exit pressure. a state that has negative process exergy destruction, that combination of variables is not feasible physically and would be immediately discarded for future consideration and further analyses. This additional insight given by the combined exergy-based approach compared to the 1st-Law only methodology provides great power when assessing performance of parametric analyses and preliminary designs. Hence, the unified exergy-based methodology has significant advantages when compared to the 1st-Law only methodology by providing a common quantifiable metric for system-level assessment as well as limiting the analysis and design space to achievable conditions.

To illustrate the systems-level component loss methodology further, consider an engine optimized for maximum thrust-specific fuel consumption (TSFC) on a sealevel thrust stand. The engine's performance is typically characterized in terms of TSFC. Assume that the engine's inlet has a perfect capture ratio and therefore no spillage of air. The engine is then nacelle-mounted on an aircraft that is designed to operate at high altitude and high speed. When the engine is operated at altitude, it does not provide the same TSFC as it did at sea level on the thrust stand. Additionally, consider that the engine may now have an imperfect capture ratio, resulting in air spillage over the nacelle to the wing. From the installed system-level performance perspective, the engine's off-design performance changes the aerodynamic performance of the wing, causing the lift and drag to change relative to the uninstalled engine configuration. The net effect is that BOTH systems no longer operate as desired or designed when viewed from a total systems perspective. However, the engine's performance is characterized in terms of TSFC and the wing's performance 
is characterized in terms of lift, drag or lift-to-drag ratio. By analogy to the simple system, a 1\% change in TSFC does not have the same impact on total system performance as a $1 \%$ change in lift. However, representing the changes in TSFC and lift or drag in terms of exergy destruction enables systemlevel comparisons to be compared consistently (common "currency"). Representing both performance parameters in the same terminology enables "apples-to-apples" comparisons for systems as well as forming the basis for componentto-system "rollups" of total losses.

\section{Concept}

Aircraft engines are generally comprised of a combination of systems, such as inlet, compressor, combustor, turbine, and nozzle (the "suck, squeeze, bang, blow" statement of propulsion). A simplified sketch of a generic turbojet engine is illustrated in Figure 1. Rather than attempt to model this complex system, the overall effects of a high energy stream coupled with a work-producing device (turbine) will serve to demonstrate the influence of losses on system performance. It is assumed that the inlet/compressor/diffuser/combustor can be modeled as a boiler producing a high energy working fluid to the turbine. The focus is therefore on how this energy is transported to the turbine and converted to useful work.

A simplified model of a portion of the turbojet system illustrated in Figure 1 was chosen to compare the differences between the 1st Law and combined 1st and 2nd Law methodologies. Modeling the combustion products as superheated steam enables variable-properties to be considered without the complexity of chemical reactions and the concomitant species equations of state. A modular calculation was used for the steam properties that permits the overall calculations to be performed semi-analytically (e.g., steam tables for the equation(s) of state coupled with the governing equations of fluid flow and thermodynamics). The duct and work-extraction device are modeled as a pipe and turbine, respectively. This system may then be used to assess the impacts of system losses (heat transfer) on turbine performance (e.g. power extraction or work rate). It is possible to employ higher-fidelity models beyond the simplified thermodynamic model used herein. Although the higher-fidelity model may be more accurate, it will not change the fundamental comparisons between the 1st and 2nd Law methodologies: the former approach may lead to notable regions in the design space that are infeasible.

3.1. Model Representation. The simplified model of the system-of-systems representing the complex engine in Figure 1 is illustrated in Figure 2. System 1 represents an energy source (compression, mixing, combustion), System 2 represents energy transfer (duct or pipe), and System 3 represents energy conversion (work-extraction via turbine device). A typical system-level loss that occurs in complex system is heat transfer (positive heat gain or negative heat loss, to or from the system resp.). In the simplified analysis presented herein, heat transfer is assumed to occur only in the pipe (i.e., all other areas are treated as adiabatic). By modeling the complex systems in a simple form, we can focus on comparing the analysis methods (i.e., 1st-Law only compared to combined 1st and 2nd Laws).

Table 1 provides additional information regarding the simplified system illustrated in Figure 2. Each component or subsystem from the actual engine is "mapped" to a simplified system that is analyzed from a 1st-Law perspective or a combined exergy-based perspective. Note that the energy source is assumed to provide a constant rate of superheated steam at a specified state to the downstream systems while the exit pressure of the turbine was fixed. These conditions provide an operating envelope for the analyses.

3.2. Test Conditions. The modeled system illustrated in Figure 2 was assumed to operate as an "installed" system, such that inlet and exit conditions were prescribed. The exit state of System 1 (superheated steam) was fixed at a pressure of 500 kilopascals $(\mathrm{kPa})$ and a temperature of $400^{\circ} \mathrm{C}$. The exit pressure for System 3 (turbine) was fixed at $7.5 \mathrm{kPa}$. These "boundary conditions" enforce a specified operating envelope on the turbine such that heat transfer (positive or negative) in System 2 will influence the performance of System 3, as illustrated qualitatively in Figure 3. Note that the amount of heat transfer must be limited by the operating envelope in this situation. In other words, it should not be possible to arbitrarily vary the heat transfer in System 2 and still meet the System 3 exit pressure constraint. Energy gain or loss in the pipe and its influence on turbine performance are analyzed in terms of 1st Law (conservation of energy) and combined 1st and 2nd Laws (exergy destruction).

Table 2 provides additional detail regarding the operating conditions for the system-of-systems. Note that high energy superheated steam simulates combustion products as the working fluid.

The system-of-systems illustrated in Figure 3 was analyzed for a number of different values of heat transfer. For discussion purposes, the test cases are more clearly presented in a matrix, as shown in Table 3 . As presented in the table, for one degree of freedom (1 DOF), only the heat transfer in System 2 was variable. Note that, a priori, the ranges of heat transfer were chosen to be representative of typical losses, but do NOT guarantee that these combinations of losses will be possible, given the constraints of the imposed operating envelope of our "installed" system.

The heat transfer from System 2 (pipe) is represented as percent of enthalpy flux entering the system. In this manner, the heat transfer is scaled consistently for each operating condition, rather than being a fixed, and possibly arbitrary, quantity. Note that heat transfer was also allowed into the system, given the fact that the system itself could possibly be housed inside, or near, another object that might be at a higher operating temperature than the system under consideration. For example, the turbine may receive energy via conduction and radiation from a nearby annular-type combustor and may also receive energy via radiation from an afterburner downstream. In either event, the possibility of heat transfer is allowed to completely represent a parametric space for analysis. 
TABLE 2: Operating conditions for Systems 1 and 3.

\begin{tabular}{lcr}
\hline Location & Thermodynamic phase & Thermodynamic state \\
\hline System 1 Exit & Super-heated steam. & $P=100 \mathrm{kPa}, T=400^{\circ} \mathrm{C}$ \\
System 3 Exit & Possible mixture of super-heated steam and saturated vapor. & $P=7 \mathrm{kPa}$. \\
\hline
\end{tabular}

Table 3: Description of test cases for 1 Degree of Freedom (DOF) and 5 cases.

\begin{tabular}{lc}
\hline Description & Range \\
\hline System 2: \% Heat transfer & -2 to 2.5 (increments of 1.25\%) \\
System 2: \% Pressure loss & 0 (frictionless) \\
System 3: \% Heat transfer & 0 (adiabatic) \\
\hline
\end{tabular}

In summary, values of heat transfer were prescribed in order to compare results when performing the analysis via standard conservation principles (1st Law only analysis) as opposed to the unified exergy-based (combined 1st and 2nd Law) methodology.

\section{Theory}

Two different analysis methodologies were performed in this study. The first method involved simultaneous solution of the continuity and energy equation coupled with the equations of state for superheated steam. This method will be referred to as 1st Law analysis (the energy equation is essentially the 1st Law of thermodynamics). The second method employed the 1st Law analysis above combined with the 2nd Law of Thermodynamics and will therefore be referred to as the combined 1st and 2nd Law analysis. The theory and analysis procedure of the two methodologies are described in this section.

4.1. 1st-Law Analysis. The 1st-Law analysis requires simultaneous solution of the continuity and energy equations along with appropriate equations of state. For the superheated steam, the online thermodynamics calculator The Expert System for Thermodynamics (TEST) was used to solve the equations of state for superheated steam [19]. Simplified forms of these governing equations are developed below.

4.1.1. Continuity Equation. On a rate basis, the integral form of the conservation of mass may be written as

$$
\frac{\partial}{\partial t} \int_{C V} \rho d V+\int_{C S} \rho \vec{V} \cdot d \vec{A}=0
$$

where

$\rho$ is fluid density,

$V$ is volume,

$\vec{V}$ is velocity,

$d \vec{A}$ is differential area element,

$(\partial / \partial t) \int_{C V} \rho d V$ is time rate of change of mass inside control volume,
$\int_{C S} \rho \vec{V} \cdot d \vec{A}=0$ is rate of mass flow across control surfaces.

Making the thermodynamic assumptions of steady state, steady flow, quasi-one dimensional flows, uniform properties at a cross section, and one inlet/one exit, (1) may be simplified to:

$$
(\rho V A)_{2}-(\rho V A)_{1}=0
$$

where the subscripts " 2 " and " 1 " represent exit and inlet, respectively. Defining the mass flow rate as

$$
\dot{m} \equiv \rho V A
$$

and substituting (3) into (2), the continuity equation may be written, for the given assumptions, as

$$
\dot{m}_{2} \equiv \dot{m}_{1}
$$

4.1.2. Energy Equation. On a rate basis, the integral form of the conservation of energy may be written as

$$
\begin{aligned}
\dot{Q}-\dot{W}_{s}-\dot{W}_{\text {shear }}-\dot{W}_{\text {other }}= & \frac{\partial}{\partial t} \int_{C V} e \rho d V \\
& +\int_{C S}(e+p v) \rho \vec{V} \cdot d \vec{A},
\end{aligned}
$$

where

$$
\begin{aligned}
& \dot{Q} \text { is heat transfer rate, } \\
& \dot{W}_{s} \text { is work rate at surface(s), } \\
& \dot{W}_{\text {shear }} \text { is work rate due to shear, } \\
& \dot{W}_{\text {other is work rate from all other forms (shaft, }} \text { electrical, nuclear, etc.), }
\end{aligned}
$$

$(\partial / \partial t) \int_{C V} e \rho d V$ is time rate of change of energy inside control volume,

$\int_{C S}(e+p v) \rho \vec{V} \cdot d \vec{A}$ flux of energy across control surface,

$e$ is specific energy $=u+(1 / 2) V^{2}+g z$,

$p$ is static pressure,

$v$ is specific volume,

$\vec{V}$ is velocity,

$\vec{A}$ is area.

Making the thermodynamic assumptions of steady state, steady flow, quasi-one dimensional flows, uniform properties at a cross section, one inlet/one exit, shear and boundary work assumed zero, turbulent flow, negligible changes in 
potential and kinetic energies compared to energy fluxes, (5) may be simplified to:

$$
\dot{Q}-\dot{W}=\rho \int_{C S}(e+p v) \vec{V} \cdot d \vec{A} .
$$

Substituting the expression for the specific energy, $e$, we have:

$$
\dot{Q}-\dot{W}=\rho \int_{C S}\left(u+\frac{V^{2}}{2}+g z+P v\right) \vec{V} \cdot d \vec{A} .
$$

Denoting inlet station by "1" and exit station by "2", and using the above assumptions and the simplified continuity, (4) and (7) may be rewritten as

$$
\begin{aligned}
\dot{Q}-\dot{W}= & \dot{m}\left[u_{2}-u_{1}\right]+\dot{m}\left[g z_{2}-g z_{1}\right]+\dot{m}\left[(P v)_{2}-(P v)_{1}\right] \\
& +\left[\int_{A_{2}}\left(\frac{1}{2} \rho V_{2}^{2}\right) V_{2} d A_{2}-\int_{A_{2}}\left(\frac{1}{2} \rho V_{1}^{2}\right) V_{1} d A_{1}\right] .
\end{aligned}
$$

Rearranging (8), introducing density for specific volume, and the definition of enthalpy $h \equiv u+P / \rho$ we get, after manipulation

$$
\begin{aligned}
\dot{Q}-\dot{W}= & \dot{m}\left[h_{2}-h_{1}\right]+\dot{m}\left[g z_{2}-g z_{1}\right] \\
& +\left[\int_{A_{2}}\left(\frac{1}{2} \rho V_{2}^{2}\right) V_{2} d A_{2}-\int_{A_{2}}\left(\rho \frac{1}{2} V_{1}^{2}\right) V_{1} d A_{1}\right] .
\end{aligned}
$$

A more practical form of the energy equation for our purposes is obtained by introducing the kinetic energy coefficient $(\beta)$, defined such that

$$
\beta \dot{m} \bar{V}^{2}=\int \rho V^{3}(d A) .
$$

Using the kinetic energy coefficient from (10) in (9), we have

$$
\begin{aligned}
\dot{Q}-\dot{W}= & \dot{m}\left[h_{2}-h_{1}\right]+\dot{m}\left[g z_{2}-g z_{1}\right] \\
& +\dot{m}\left[\left(\frac{1}{2} \beta_{2} \bar{V}_{2}^{2}\right)-\left(\frac{1}{2} \beta_{1} \bar{V}_{1}^{2}\right)\right] .
\end{aligned}
$$

For the vast majority of turbulent, high Reynolds number flows, the kinetic energy coefficient $(\beta)$ is nearly unity. Making this assumption yields

$$
\begin{aligned}
\dot{Q}-\dot{W}= & \dot{m}\left[h_{2}-h_{1}\right]+\dot{m}\left[\left(g z_{2}\right)-\left(g z_{1}\right)\right] \\
& +\dot{m}\left[\left(\frac{1}{2} \bar{V}_{2}^{2}\right)-\left(\frac{1}{2} \bar{V}_{1}^{2}\right)\right] .
\end{aligned}
$$

In the present situation, the "flow" rate of energy (as represented by the mass flow rate and enthalpy product) through the system is much larger than the changes in potential and kinetic energies. Making this last assumption and rearranging, the energy equation becomes:

$$
\dot{Q}-\dot{W}-\dot{m}\left[h_{2}-h_{1}\right]=0 .
$$

4.1.3. Equations of State. The state postulate of thermodynamics stipulates that any two independent intensive thermodynamic properties fully specify the state for a pure substance. In other words, if temperature and pressure are known, the enthalpy is determined. Similarly, temperature and specific entropy may also be used to determine the enthalpy. Written in general, we may write the equations of state as

$$
h=h(T, P) \quad \text { or } h=h(T, s), \quad \ldots
$$

The 1st-Law analysis required the simultaneous solution of the simplified continuity and energy equations, together also with the equation(s) of state, as given by (4), (13), and (14), respectively, for the variations in heat transfer listed in Table 3.

4.2. Combined 1st and 2nd Law: Exergy-Based Analysis. The combined 1st and 2nd Law analysis requires simultaneous solution of the continuity and exergy equations along with appropriate equations of state. The continuity equation was developed in the previous section and is not repeated here. Simplified forms of the remaining governing equations are developed below.

4.2.1. Entropy Balance (2nd Law). For a control volume, on a rate basis, the balance of entropy may be written:

$$
\int_{\text {path }}\left(\frac{\delta \dot{Q}}{T}\right)+\int_{C S} s \rho \vec{V} \cdot(d \vec{A})+\dot{S}_{\text {gen }}=\frac{d S_{C V}}{d t},
$$

where

$T$ is temperature of surface where heat transfer occurs,

$\delta \dot{Q}$ is rate of path-dependent heat transfer across surface(s),

$s$ is specific entropy,

$\rho$ is density,

$\vec{V}$ is velocity vector,

$d \vec{A}$ is area vector,

$\dot{S}_{\text {gen }}$ is rate of entropy generation,

$d S_{C V} / d t$ is rate of change of entropy in the control volume.

Making the thermodynamic assumptions of quasi-one dimensional flows, uniform properties at a cross section, and one inlet/one exit, (15) may be simplified to:

$$
\int_{\text {path }}\left(\frac{\delta \dot{Q}}{T}\right)+[(\rho \bar{V} A) s]_{1}-[(\rho \bar{V} A) s]_{2}+\dot{S}_{\text {gen }}=\frac{d S_{C V}}{d t},
$$

where the subscripts "1" and "2" refer to inlet and exit, respectively. Introducing the continuity (4) and assuming 
uniform heat transfer rate at a boundary " $k$ " and steady flow, (16) becomes

$$
\sum_{k=1}^{n}\left(\frac{\dot{Q}_{k}}{T_{k}}\right)+\dot{m}\left(s_{1}-s_{2}\right)+\dot{S}_{\mathrm{gen}}=0
$$

The formulation of the entropy balance, (16), states that the rate of transfer of entropy (first term) and the net flow rate of entropy (second term) must be balanced by the entropy generation rate (third term).

4.2.2. Exergy Balance (Combined 1st and 2nd Laws). For a control volume, on a rate basis, the balance of exergy may be written as

$$
\begin{aligned}
& \int_{C S}\left(1-\frac{T_{0}}{T_{k}}\right) \delta \dot{Q}-\left(\dot{W}-P_{0} \frac{d \forall_{C V}}{d t}\right) \\
& +\int_{C S} \psi \rho \vec{V} \cdot(d \vec{A})-\dot{X}_{\text {Destroyed }}=\frac{d X_{C V}}{d t},
\end{aligned}
$$

$T_{k}$ is temperature of " $k$ th" surface,

$T_{0}$ is reference temperature (dead state, absolute units),

$\delta \dot{Q}$ is rate of path-dependent heat transfer across surfaces,

$\dot{W}$ is work rate across boundary,

$P_{0}$ is reference pressure (dead state, absolute units),

$d \forall_{C V} / d t$ is rate of change of volume for control volume,

$\psi$ is exergy per mass; $\psi \equiv\left(h-h_{0}\right)+g z+\left(\bar{V}^{2} / 2\right)-$ $T_{0}\left(s-s_{0}\right)$,

$\vec{V}$ is velocity vector,

$d \vec{A}$ is area vector increment,

$\dot{X}_{\text {Destroyea }}$ is Rate of exergy destruction; $\dot{X}_{\text {Destroyed }}=$ $T_{0} \dot{S}_{\text {gen }}$,

$d \mathrm{X}_{C V} / d t$ is rate of change of exergy in the control volume.

Making the thermodynamic assumptions of quasi-one dimensional flows, uniform properties at a cross section, steady flow, and performing the vector dot product, the exergy balance (18) may be simplified to

$$
\begin{aligned}
& \int_{C S}\left(1-\frac{T_{0}}{T_{k}}\right) \delta \dot{Q}-\left(\dot{W}-P_{0} \frac{d V_{C V}}{d t}\right)+\sum_{\text {Inlets }}(\dot{m} \psi) \\
& -\sum_{\text {Exits }}(\dot{m} \psi)-\dot{X}_{\text {Destroyed }}=0 .
\end{aligned}
$$

For one inlet, one exit, uniform heat transfer at each surface and using continuity, (19) may be further simplified to

$$
\begin{array}{r}
\sum_{k=1}^{n}\left(1-\frac{T_{0}}{T_{k}}\right) \dot{Q}_{k}-\left(\dot{W}-P_{0} \frac{d V_{C V}}{d t}\right) \\
+\dot{m}\left(\psi_{1}-\psi_{2}\right)-\dot{X}_{\text {Destroyed }}=0 .
\end{array}
$$

For many flow situations (flow through ducts, nozzles, diffusers, etc.), the boundaries remain fixed, and therefore $d \forall_{C V} / d t=0$. For such cases, (20) may be further simplified to

$$
\sum_{k=1}^{n}\left(1-\frac{T_{0}}{T_{k}}\right) \dot{Q}_{k}-\dot{W}+\dot{m}\left(\psi_{1}-\psi_{2}\right)-\dot{X}_{\text {Destroyed }}=0 .
$$

Further, if there is only one isothermal surface (subscript " $b$ " for boundary) exchanging thermal energy, we get:

$$
\left(1-\frac{T_{0}}{T_{b}}\right) \dot{Q}_{b}-\dot{W}-\dot{X}_{\text {Destroyed }}+\dot{m}\left(\psi_{1}-\psi_{2}\right)=0 .
$$

Substituting the expressions for specific exergy and exergy destruction rate and ignoring changes in potential and kinetic energies' we may write (22) as

$$
\begin{aligned}
& \left(1-\frac{T_{0}}{T_{b}}\right) \dot{Q}_{b}-\dot{W}-T_{0} \dot{S}_{\text {gen }} \\
& \quad+\dot{m}\left(h_{1}-h_{2}\right)-\dot{m} T_{0}\left(s_{1}-s_{2}\right)=0,
\end{aligned}
$$

where the subscripts " 1 " and " 2 " refer to inlet and exit, respectively. Rearranging (23) into a form similar to the energy equation yields:

$$
\left(1-\frac{T_{0}}{T_{b}}\right) \quad \dot{Q}_{b}-\dot{W}+\dot{m}\left(h_{1}-h_{2}\right)=T_{0} \dot{S}_{g e n}+\dot{m} T_{0}\left(s_{1}-s_{2}\right) .
$$

The energy balance, (13), is rewritten here for comparison as

$$
\dot{Q}-\dot{W}-\dot{m}\left[h_{2}-h_{1}\right]=0 .
$$

Note that if the dead state temperature $T_{0}$ is small compared to the boundary temperature $T_{b}$, the comparison between the energy balance (1st Law), (25) and the exergy balance, (24), becomes much clearer. The exergy balance equation represents a combination of the 1st and 2nd Laws because it is essentially the conservation of energy (1st Law) constrained by entropy production (the 2nd Law). In fact, rearranging (24) to reflect that entropy generation must be greater than or equal to zero provides

$$
\begin{aligned}
T_{0} \dot{S}_{\text {gen }}= & \left(1-\frac{T_{0}}{T_{b}}\right) \dot{Q}_{b}-\dot{W}+\dot{m}\left(h_{1}-h_{2}\right) \\
& -T_{0} \dot{m}\left(s_{1}-s_{2}\right) \geq 0
\end{aligned}
$$

The left hand side of (26) represents the exergy destruction rate, the so-called Guoy-Stodola theorem [10].

The 1st Law analysis performed in this study solved simultaneously the simplified continuity and energy balance, (4) and (13), respectively (with appropriate equations of state). Likewise, for the combined exergy-based analysis, we solved simultaneously the simplified continuity and exergy balance equations (4) and (25), respectively (with appropriate equations of state). The results of these calculations for parametric variations in heat transfer listed in Table 3 are presented in the next section. 


\section{Results}

The results for the 1 degree-of-freedom (1 DOF) test cases shown in Table 3 and analyzed via the 1st Law as well as the combined 1st and 2nd Laws are presented in this section. Note that the 1st Law analysis does not constrain the direction of energy transfer nor does it enforce the nonnegative entropy production. These are both significant shortcomings for the 1st Law analysis in that the 1st Law analysis provides no guidance or bounds as to whether or not a particular state may exist or a process may occur. As such, the 1st Law admits physically impossible solutions in the analysis or design space. This is an important distinction between the two different methodologies in that the combined 1st and 2nd Law analysis accounts for the conservation of mass and energy but also provides constraints on which solutions are physically possible. These restrictions are especially true when an operating envelope is specified for the system (System 1 exit conditions fixed and System 3 exit pressure fixed). Therefore, there are many situations where the results of the 1st Law analysis, although satisfying the basic conservation laws of mass and energy, are NOT physically possible and cannot occur within the operating envelope.

Note 2. The philosophy employed in our parametric analysis followed a "what-if" approach, akin to preliminary design without a priori knowledge of the results. In other words, from a baseline perspective, what would be the result if the System 2 heat transfer increased or decreased by some percentage?

5.1. One Degree of Freedom (1 DOF). For the one degree-offreedom (1 DOF) cases, only heat transfer in System 2 (the transfer pipe) was allowed. Consistent with thermodynamic convention, positive heat transfer is assumed to be into the system (gain, or numerically positive) while negative heat transfer is assumed to be out of the system (loss, or numerically negative).

5.1.1. 1 DOF, 1st Law Analysis. The results of the 1st Law analysis of the parametric combinations of heat transfer for the 1 DOF cases are presented in Table 4 and illustrated in Figure 4. Note that the heat transfer has a direct linear relationship on turbine power extraction for the $1 \mathrm{DOF}$ case. This is intuitively obvious, as more energy is available to the turbine because energy is added in the pipe via positive heat transfer. In fact, according to 1 st Law analysis for the cases performed, the maximum power produced in the turbine occurs at the maximum positive heat transfer in System 2 ( $409 \mathrm{~kW}$ of heat gain in the pipe yields $4435 \mathrm{~kW}$ turbine power). Conversely, according to 1 st Law analysis, the minimum power extracted from the turbine occurs at the maximum negative heat transfer in System $2(409 \mathrm{~kW}$ of heat loss in the pipe yields $3992 \mathrm{~kW}$ turbine power).

The results presented in Table 4 and Figure 4 suggest that adding energy in System 2 increases turbine performance. This is in fact true from a 1st Law-only perspective, wherein no consideration is made about the physical possibility of doing so. In order to answer that question, one must
TABLE 4: Numerical results for 1 DOF 1st law analysis.

\begin{tabular}{|c|c|c|c|c|c|c|}
\hline \multicolumn{2}{|c|}{ System 2 (Pipe) } & \multicolumn{2}{|c|}{ System 2(Pipe) } & \multicolumn{2}{|c|}{ System 3 (Turbine) } & \multirow{3}{*}{$\begin{array}{c}\text { Turbine } \\
\text { Power } \\
k W\end{array}$} \\
\hline \multicolumn{2}{|c|}{ Heat transfer } & \multicolumn{2}{|c|}{ Pressure drop } & \multicolumn{2}{|c|}{ Heat transfer } & \\
\hline$\%$ & $k W$ & $\%$ & $k P a$ & $\%$ & $k W$ & \\
\hline 2.50 & 408.98 & 0 & 0 & 0 & 0 & 4434.93 \\
\hline 1.25 & 204.49 & 0 & 0 & 0 & 0 & 4319.18 \\
\hline 0.00 & 0.00 & 0 & 0 & 0 & 0 & 4203.44 \\
\hline-1.25 & -204.49 & 0 & 0 & 0 & 0 & 4097.95 \\
\hline-2.50 & -408.98 & 0 & 0 & 0 & 0 & 3992.46 \\
\hline
\end{tabular}

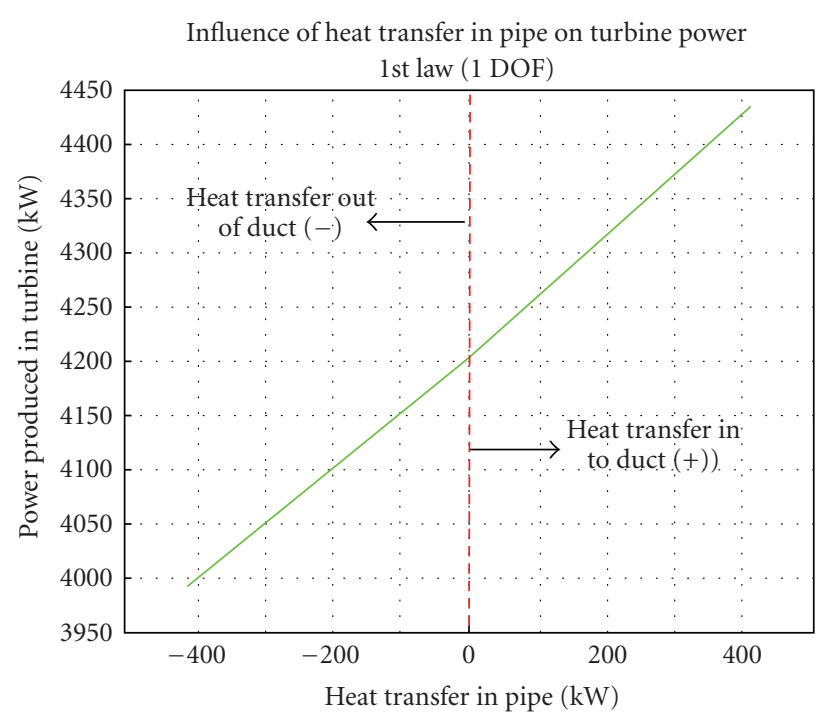

FIGURE 4: Graphical results for 1 DOF 1st Law Analysis. Increasing heat transfer results in increased turbine performance.

consider the 2nd Law directionality in addition to the 1st Law quantification (i.e. conservation of energy). When the 1st and 2nd Laws are combined in the analysis, the addition of energy in System 2 is constrained by the fact that the exergy destruction must be greater than or equal to zero. Therefore, although it may be desirable to add energy in System 2, it may not be achievable from a physical point of view and still operate under the given conditions (i.e., the solution, although numerically consistent with the conservation laws of continuity and energy, does not represent a feasible physical solution).

5.1.2. 1 DOF, Combined 1st and 2nd Laws. The results of the combined 1st and 2nd Law analysis for the 1 DOF cases are presented in Table 5. Heat transfer rate is plotted against exergy destruction in Figure 5. The relationship between heat transfer and exergy destruction is clear: the minimum nonnegative exergy destruction occurs for the adiabatic case. Because negative exergy destruction violates the 2nd Law, all positive values of heat transfer in System 2 (for the given conditions) are not possible. This has important ramifications for turbine performance in this case. From Table 4, it can be seen that the 1st Law analysis provided a maximum turbine performance of $4435 \mathrm{~kW}$ at $+409 \mathrm{~kW}$ of 


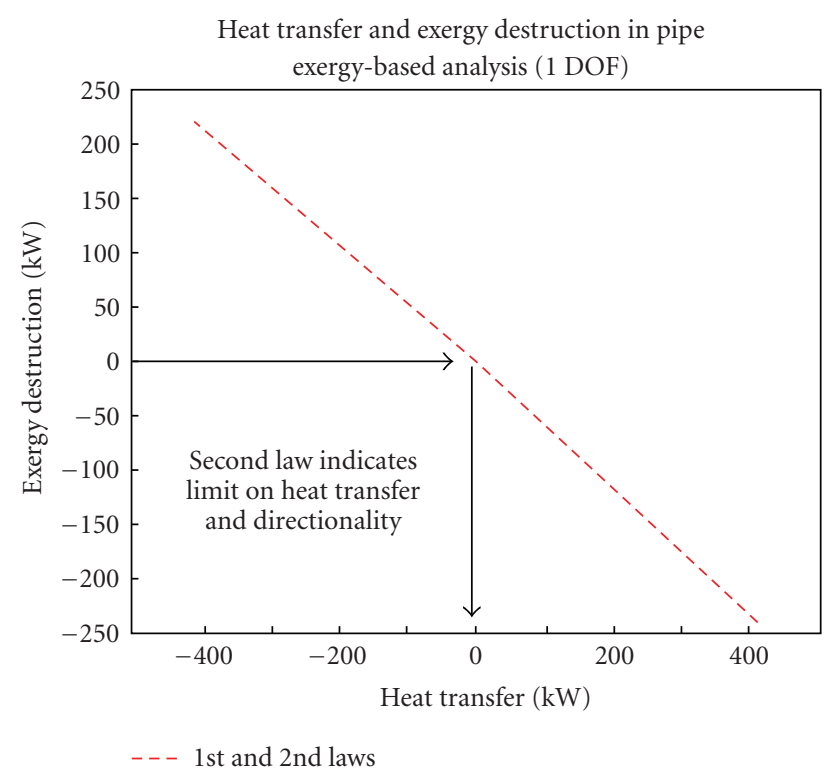

Figure 5: Relationship between heat transfer and exergy destruction in System 2. Higher heat loss from System 2 results in greater exergy destruction. Note that only nonnegative exergy destruction is allowed and therefore heat gain in System 2 is not possible.

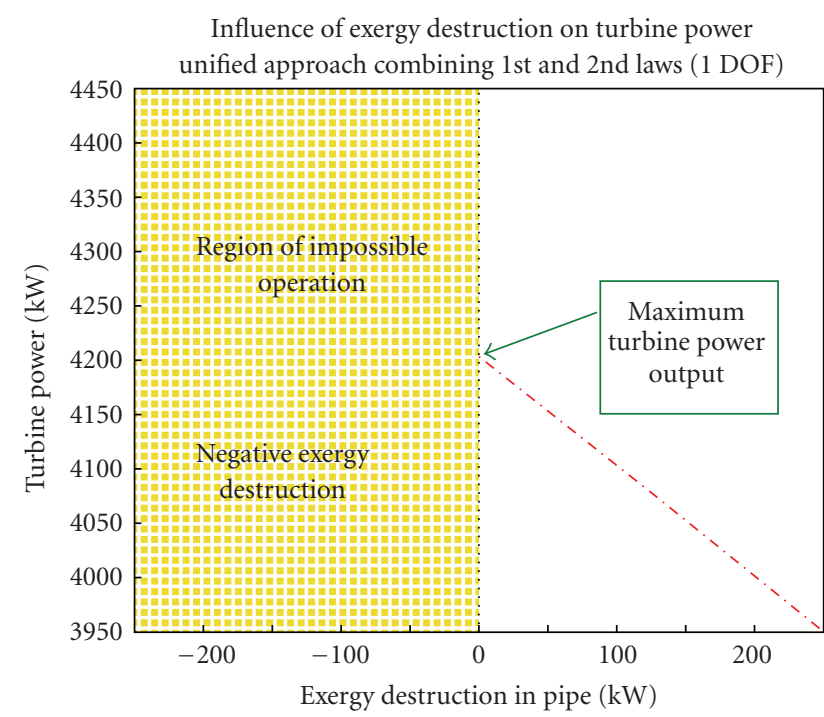

FIGURE 6: Graphical results of 1 DOF 1st and 2nd Law Analysis. Increasing exergy destruction results in reduced turbine performance. Note that only nonnegative exergy destruction is allowed.

heat transfer in the pipe. However, the 2nd Law restriction of nonnegative exergy destruction dictates that a maximum possible turbine performance of $4203 \mathrm{~kW}$ occurs at the adiabatic condition.

Combining the 2nd Law with the 1st Law indicates that there is a physical limit for heat transfer in System 2 as imposed by nonnegative exergy destruction. Therefore, as seen in Table 5, although it is desirable to add energy in System 2 via heat transfer (turbine power increases with increasing heat transfer), it is impossible to provide positive heat transfer in this situation as this results in negative exergy destruction (violates the 2 nd Law). Comparing this maximum achievable performance of $4203 \mathrm{~kW}$ (combined 1st and 2nd Laws), to the maximum calculated performance of $4435 \mathrm{~kW}$ (1st Law), it can be seen that the 1st Law over-predicts the maximum turbine performance by approximately $5.5 \%$. Additionally, and perhaps more importantly, note that 2 of the 5 cases $(40 \%)$ in Table 5 result in negative exergy destruction. This means that these results are not valid and that further analysis and assessment should not be considered. This is important in defining a "roadmap" for advanced parametric analyses and/or optimization schema in that the analyses may be performed more efficiently by eliminating many cases that are not feasible.

The exergy-based analysis provides additional insight that is not possible with the 1st Law alone. In order to see this point, refer again to the data presented in Table 5. Note that 1st Law analyses are still interpretable from a heat transfer perspective (i.e., increasing heat transfer increases turbine performance). Further, the 1st Law results are valid for negative heat transfer up to the limiting point of the adiabatic condition, which is established by the 2 nd Law. From Table 5, note that all values of turbine power that result from positive heat transfer are not achievable as they violate the 2nd Law by producing negative exergy destruction. These non-physically-possible results are labeled "Region of impossible operation" in Figure 6. The additional insights from the combined 1st and 2nd Laws are therefore seen to be twofold. First, the combined 1st and 2nd Laws establish physical limits for achievable conditions by clearly noting regions of impossible operation that result from negative exergy destruction. Second, the combined 1st and 2nd Laws clearly illustrate an upper limit for performance as the point of minimum exergy destruction (yellow highlighted entry in Table 5). Both of these insights are useful in limiting the parametric space to be analyzed as well as form the basis for optimizations from a systems-level perspective.

\section{Summary}

We presented the development and comparison of two different methodologies, the 1st-Law only (conventional approach) and a combined 1st and 2nd Laws (exergy-based approach) for the analysis of interacting systems. A portion of a complex aircraft engine system was modeled in a simple manner in order to demonstrate the differences in the two methodologies. This simplified model was set up to illustrate the methodology. It may be observed that the result was intuitive since nothing was modeled to add energy to System 2. At the same time, the method quantitatively shows that minimizing the thermal energy lost from System 2 is beneficial. This could be used to design insulation to achieve improved performance, for example. The relevant governing equations were solved for one, two, and three system degreesof-freedom (1 DOF, 2 DOF, and 3 DOF, resp.). However, only the 1 DOF cases were presented herein for clarity; we plan to present the additional DOF cases in a subsequent report. 
TABLE 5: Numerical results for 1 DOF exergy-based analysis.

\begin{tabular}{|c|c|c|c|c|c|c|c|}
\hline \multicolumn{2}{|c|}{ System 2 (Pipe) } & \multicolumn{2}{|c|}{ System 2(Pipe) } & \multirow{3}{*}{$\begin{array}{c}\text { System } 2 \text { (Pipe) } \\
\text { Exergy destruction } \\
k W\end{array}$} & \multicolumn{2}{|c|}{ System 3 (Turbine) } & \multirow{3}{*}{$\begin{array}{c}\text { Turbine } \\
\text { Power } \\
k W\end{array}$} \\
\hline \multicolumn{2}{|c|}{ Heat transfer } & \multicolumn{2}{|c|}{ Pressure drop } & & & & \\
\hline$\%$ & $k W$ & $\%$ & $k P a$ & & $\%$ & $k W$ & \\
\hline 2.50 & 408.98 & 0 & 0 & -240.14 & 0 & 0 & 4434.93 \\
\hline 1.25 & 204.49 & 0 & 0 & -120.08 & 0 & 0 & 4319.18 \\
\hline 0.00 & 0.00 & 0 & 0 & 0.00 & 0 & 0 & 4203.44 \\
\hline-1.25 & -204.49 & 0 & 0 & 110.31 & 0 & 0 & 4097.95 \\
\hline-2.50 & -408.98 & 0 & 0 & 220.64 & 0 & 0 & 3992.46 \\
\hline
\end{tabular}

\section{Conclusions}

The major purpose of this overall work has been to assess the difference in results of system performance when using the 1st Law only (conservation of energy) compared to the combined 1st and 2nd Laws (exergy destruction). The development and results clearly indicate that both analyses are related. In fact, the 1st Law (focus on conservation of energy) can be recast in terms of the combined 1st and 2nd Laws (focus on exergy destruction). It is important to note that exergy-destruction analysis is not "reinventing the wheel", it is merely interpreting the results differently. Using a simpler thermodynamic model for this study, it was clearly demonstrated that the 1st Law approach is only a necessary but not sufficient condition for preliminary design applications. The sufficiency condition for design requires the additional consideration of feasibility provided by the 2nd Law.

The combined 1st and 2nd Law analysis has significant advantages over the 1st Law analysis. First, the combined 1st and 2nd Law analysis provides physical limits on performance that the 1st Law analysis does not. This is crucial to note when performing fundamental research or preliminary design (either parametrically or employing an optimization schema). This point is illustrated by the fact that, for the 1 degree-of-freedom results and the conditions analyzed, $40 \%$ of the results obtained from the 1st Law analysis were not feasible. This has far-reaching implications in that, if the results from a 1st Law analysis are used for establishing operating conditions and design parameters, many of the apparently best operating conditions can never be achieved. Furthermore, when analyzing large parametric combinations of variables in a 1st Law analysis, it is likely that many of the conditions analyzed are not feasible, resulting in wasted effort and possibly improper interpretation (i.e., the wrong conclusions). Third, when multiple systems are combined into a system-of-systems approach, the combined 1st and 2nd Laws (exergy destruction focus) provides a consistent accounting for all forms of losses regardless of point of origin (e.g., System 2 or System 3) and/or source of irreversibility (e.g., heat transfer, friction, etc.). This point illustrates that the exergy destruction concept is easily extensible from any individual component to a complex system, all with consistent interpretation.

Taken in total, the 1st Law only approach has serious deficiencies compared to the combined 1st and 2nd Law approach. We suggest that the conventional approach using only the 1st Law should neither be rigorously defended nor employed in a complex system-of-systems approach. In fact, the combined 1st and 2nd Laws should be adopted for all analyses and optimizations of systems involving irreversibilities, especially in systems with large uncertainties in performance (e.g., research and design efforts where legacy systems and experience do not exist). Therefore, we strongly recommend that the system analysis and design paradigm be shifted from the 1st Lawonly conventional approach to the combined 1st and 2nd Law exergy-based methodology. The 1st Law approach is interpreted as providing the necessary conditions for analysis and design (i.e., obeying the conservation equations), whereas the combined 1st and 2nd Law approach provides the necessary and sufficient conditions (i.e., obeying the conservation equations in feasible space).

\section{Nomenclature}

$\begin{array}{ll}A: & \text { Area } \\ d \vec{A}: & \text { Area element vector } \\ e: & \text { Specific energy } \\ g: & \text { Gravitational acceleration } \\ h: & \text { Specific enthalpy; } h \equiv u+P / \rho \\ h_{0}: & \text { Reference specific enthalpy (dead state) } \\ \dot{m}: & \text { Mass flow rate } \\ P: & \text { Pressure } \\ P_{0}: & \text { Reference pressure (dead state) } \\ \dot{Q}: & \text { Heat transfer rate } \\ \delta \dot{Q}: & \text { Rate of path-dependent heat transfer across } \\ & \text { surface } \\ s: & \text { Specific entropy } \\ s_{0}: & \text { Reference specific entropy (dead state) } \\ S: & \text { Extensive entropy } \\ \dot{S_{\text {gen }}}: & \text { Entropy generation rate } \\ t: & \text { Time } \\ T: & \text { Temperature } \\ T_{k}: & \text { Temperature of "kth" surface } \\ T_{0}: & \text { Reference temperature (dead state) } \\ u: & \text { Specific internal energy } \\ v: & \text { Specific volume } \\ \vec{V}: & \text { Velocity } \\ \forall: & \text { Volume } \\ \dot{W}_{\text {other }}: & \text { Work rate from all other forms (shaft } \\ \dot{W} & \text { electrical, nuclear, etc.) } \\ \text { shear }: & \text { Work rate due to shear }\end{array}$


$\dot{W}_{s}$ : Work rate at surface(s)

$z$ : Relative height for potential energy.

\section{Subscripts}

1: Inlet flow station

2: Exit flow station

0: $\quad$ Reference dead state for exergy

$b$ : Boundary

CS: Control Surface

$C V$ : Control Volume

gen: Generation

$k$ : "k"th item.

\section{Greek Symbols}

$\beta$ : Kinetic energy coefficient

$\psi$ : Flow specific exergy

$\rho$ : Fluid density.

\section{Acknowledgments}

The authors gratefully acknowledge the support of the US Air Force Office of Scientific Research under the program management of Dr. Victor Giurgiutiu (Structural Mechanics) and the US Air Force Research Laboratory, Multidisciplinary Technologies Center, for funding this research. This material is declared a work of the U.S. Government and not subject to copyright protection in the United States.

\section{References}

[1] D. J. Moorhouse, "The multidisciplinary engineer in the context of concurrent engineering," in Aerodynamic Design and Optimization of Flight Vehicles in a Concurrent MultiDisciplinary Environment, June 2000, RTO Meeting Proceedings, RTO-MP-35.

[2] D. J. Moorhouse, "Proposed system-level multidisciplinary analysis technique based on exergy methods," Journal of Aircraft, vol. 40, no. 1, pp. 11-15, 2003.

[3] K. Alabi, et al., "Multi-level exergy modeling, analysis and optimization for the integrated synthesis/design of aerospace systems," in Proceedings of the 25th Congress of the International Council of the Aeronautical Sciences (ICAS '06), Hamburg, Germany, September 2006, ICAS paper 1.9.2.

[4] V. Periannan, M. R. von Spakovsky, and D. J. Moorhouse, "Investigation of the effects of various energy and exergybased figures of merit on the optimal design of a high performance aircraft system," in Proceedings of ASME International Mechanical Engineering Congress and Exposition (IMECE '06), Chicago, Ill, USA, November 2006.

[5] J. A. Camberos, "On the construction of entropy balance equations for arbitrary thermophysical processes," in Proceedings of the 39th AIAA Aerospace Sciences Meeting, Reno, Nev, USA, January 2001, AIAA paper no. 2001-0815.

[6] J. A. Camberos, "Entropy concavity, the second-law, and the concept of exergy in thermophysics," in Proceedings of the 33rd AIAA Thermophysics Conference, Norfolk, Va, USA, June 2000, AIAA no. paper 99-3557.
[7] Y. A. Cengel and M. A. Boles, Thermodynamics: An Engineering Approach, McGraw Hill, New York, NY, USA, 6th edition, 2007.

[8] R. W. Fox, A. T. McDonald, and P. J. Pritchard, Introduction to Fluid Mechanics, John Wiley \& Sons, New York, NY, USA, 6th edition, 2004.

[9] A. Bejan, et al., Thermal Design and Optimization, John Wiley \& Sons, New York, NY, USA, 1996.

[10] A. Bejan, Advanced Engineering Thermodynamics, John Wiley \& Sons, New York, NY, USA, 1997.

[11] A. Bejan, Entropy Generation Minimization, CRC Press, New York, NY, USA, 1996.

[12] D. W. Riggins, C. R. McClinton, and P. H. Vitt, "Thrust losses in hypersonic engines part 1: methodology," Journal of Propulsion and Power, vol. 13, no. 2, pp. 281-287, 1997.

[13] D. W. Riggins, "Thrust losses in hypersonic engines part 2: applications," Journal of Propulsion and Power, vol. 13, no. 2, pp. 288-295, 1997.

[14] D. W. Riggins, "Evaluation of performance loss methods for high-speed engines and engine components," Journal of Propulsion and Power, vol. 13, no. 2, pp. 296-304, 1997.

[15] J. R. Muñoz and M. R. von Spakovsky, "A decomposition approach for the large scale synthesis/design optimization of highly coupled, highly dynamic energy systems," International Journal of Applied Thermodynamics, vol. 4, no. 1, pp. 19-33, 2001.

[16] V. Periannan, Investigation of the effects of different objective functions/figures of merit on the analysis and optimization of high performance aircraft system synthesis/design, M.S. thesis, Virginia Polytechnic Institute and State University, Blacksburg, Va, USA, 2005.

[17] D. F. Rancruel and M. R. von Spakovsky, "Use of a unique decomposition strategy for the optimal synthesis/design and operation of an advanced fighter aircraft system," in Proceedings of the 10th AIAA/ISSMO Multi-Disciplinary Analysis and Optimization Conference, Albany, NY, USA, August-September 2004.

[18] T. M. Taylor, A. Khamooshi, and D. W. Riggins, "Innovative concepts for large-scale drag and heat transfer reductions in high-speed flows," in Proceedings of the 44th AIAA Aerospace Sciences Meeting, vol. 11, pp. 7850-7871, Reno, Nev, USA, January 2006.

[19] S. Battacharjee, "The Expert System for Thermodynamics (TEST), (Professional Version)," San Diego State University, 2007 http://energy.sdsu.edu/testcenter/. 

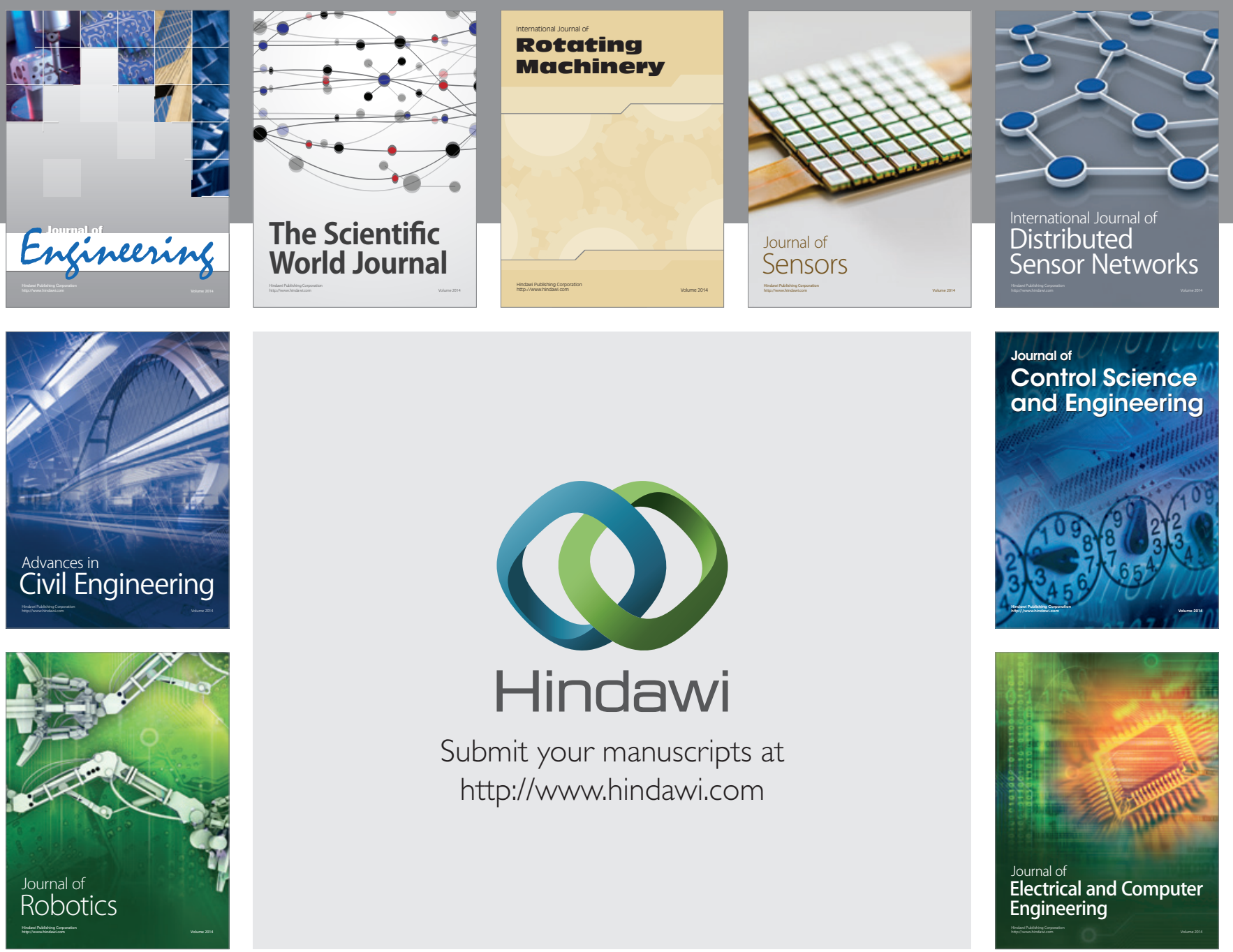

Submit your manuscripts at

http://www.hindawi.com
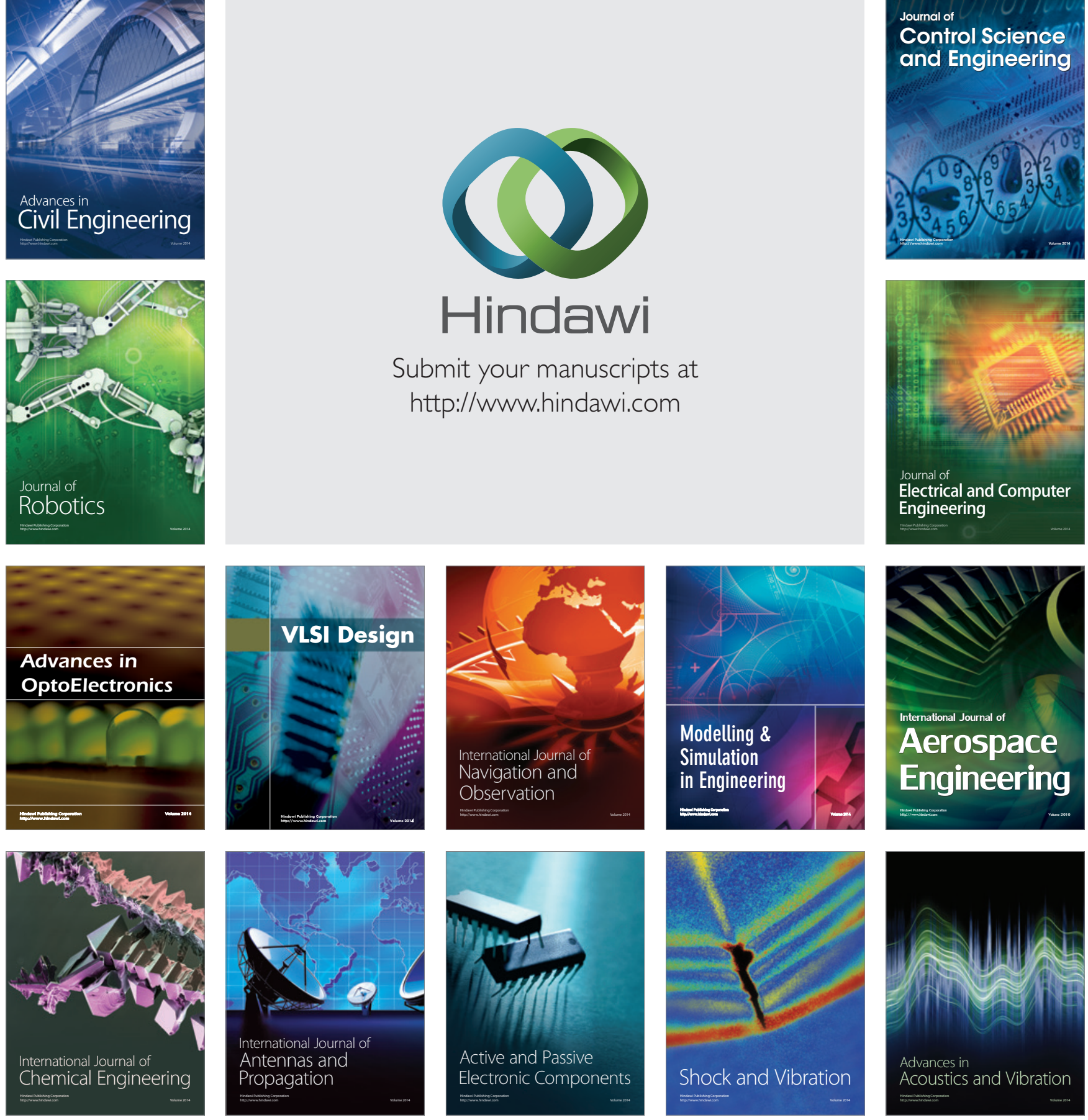